\title{
SHARP: Spectrum Harvesting with ARQ Retransmission and Probing in Cognitive Radio
}

\author{
James C. F. Li, Member, IEEE, Wei Zhang, Senior Member, IEEE, Aria Nosratinia, Fellow, IEEE, \\ and Jinhong Yuan, Senior Member, IEEE
}

\begin{abstract}
In underlay cognitive radio, a secondary user transmits in the transmission band of a primary user without serious degradation in the performance of the primary user. This paper proposes a method of underlay cognitive radio where the secondary pair listens to the primary ARQ feedback to glean information about the primary channel. The secondary transmitter may also probe the channel by transmitting a packet and listening to the primary $A R Q$, thus getting additional information about the relative strength of the cross channel and primary channel. The method is entitled Spectrum Harvesting with ARQ Retransmission and Probing (SHARP). The probing is done only infrequently to minimize its impact on the primary throughput. Two varieties of spectrum sharing, named conservative and aggressive SHARP, are introduced. Both methods avoid introducing any outage in the primary; their difference is that conservative SHARP leaves the primary operations altogether unaffected, while aggressive SHARP may occasionally force the primary to use two instead of one transmission cycle for a packet, in order to harvest a better throughput for the secondary. The performance of the proposed system is analyzed and it is shown that the secondary throughput can be significantly improved via the proposed approach, possibly with a small loss of the primary throughput during the transmission as well as probing period.
\end{abstract}

Index Terms-ARQ, cognitive radio, outage probability, spectrum sharing.

\section{INTRODUCTION}

$\mathbf{T}$ HERE exists a rich literature on the coexistence of primary and secondary users in the same time/frequency band. Gastpar [1] formulates the capacity problem under the power constraints at the receiver side in AWGN channel, which mimics the basic structure of the cognitive radio networks. Furthermore, Ghasemi and Sousa [2] extend [1] to the ergodic capacity in fading channels (e.g. Rayleigh, Nakagami) with peak or average interference constraint on the primary receiver. Ben Letaief and Zhang [3] combine cooperation with cognition and propose robust cooperative spectrum sensing techniques for a practical framework. Zhang et al. [4] finds

Manuscript received July 18, 2011; revised March 9 and September 6, 2012. The associate editor coordinating the review of this paper and approving it for publication was S. Roy.

J. C. F. Li is with NEC Laboratories China, Beijing China (e-mail: jamescfli@ieee.org). He was with the School of Electrical Engineering and Telecommunications, The University of New South Wales, Sydney, Australia.

W. Zhang and J. Yuan are with the School of Electrical Engineering and Telecommunications, The University of New South Wales, Sydney, Australia (e-mail: wzhang@ee.unsw.edu.au, j.yuan@unsw.edu.au). Their work was supported in part by the Australian Research Council Discovery Project DP0987944.

A. Nosratinia is with the Department of Electrical Engineering, University of Texas at Dallas, Richardson, TX 75080, USA (e-mail: aria@utdallas.edu). His research was in part supported by THECB via grant 009741-0084-2007. Digital Object Identifier 10.1109/TCOMM.2013.13.110471 that the cognitive users can exploit intermediate nodes to relay the information when there is no common white frequency band connecting the source and the destination. In [5], [6], three kinds of transmission schemes, namely underlay, overlay and interweave, are introduced.

In order to limit the interference on the primary, the secondary should judiciously adapt its power based on the Channel State Information (CSI) of the primary channel. However, this is not straight forward as the secondary does not have direct access to the primary CSI. In [7], [8], a secondary system is allowed to transmit in the primary frequency band and the control feedback, such as ARQ, channel state, or power control information, from the primary serves as the side information to the secondary terminals. Similarly, in [9], [10] the secondary user co-exists with a maximal- $T$-transmission primary system, under a constraint on the maximum throughput loss of the primary; [11] and [12] investigate techniques for exploiting the redundancy of the primary transmission process, as introduced by the ARQ scheme, i.e. the same copies of messages transmitted in subsequent time slots. In addition, [13] notices a hidden feedback loop between the primary and the secondary, and proposes a supervised transmission scheme to effectively share the primary spectrum. Zhang et al. [14] assumes that the primary is a two-way communication system and the so-called effective interference channel is estimated through the primary signals. Huang et al. [15] addresses a framework where multiple secondaries eavesdrop on the 1-bit primary control information to update their transmit power in a distributed fashion.

Tannious and Nosratinia [16] propose to use the ARQ feedback information to harvest excess mutual information in the channel when the primary has constant rate and power, while the channel gains fluctuate due to fading. The essence of the idea of [16] is that whenever the primary receiver sends a NACK, other nodes in the system (potentially) become aware that a second transmission will be underway. Because the first transmission has already provided some information to the primary receiver (albeit not enough), the second transmission now needs to provide less than a full amount of mutual information and can be more robust to interference.

The system model in [16] was based on uncorrelated (i.i.d.) channel gains across time. In the present paper, we consider a system model where the channel gains are no longer i.i.d., but rather, the channel experiences slow fading that extends through several transmission intervals. This system model applies to mobiles that are in an essentially stationary environment, e.g., ad-hoc networks in an office environment. 


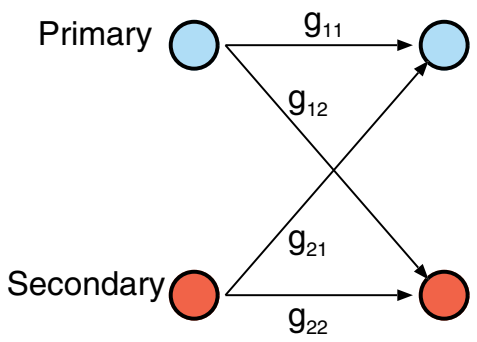

Fig. 1. ARQ-based spectrum sharing without CSI at secondary transmitter.

We consider two additional opportunities in this scenario: (1) each primary ARQ now carries information about the channel gains in multiple time intervals, and (2) each secondary transmission creates interference on the primary, therefore the following ARQ carries further information back to the secondary regarding the relative strength of primary and cross channel coefficients. In effect, this latter source of information can be thought of as a probing of the primary channel by the secondary, whose details will be made clear in the sequel. Note that both these additional information sources are a result of the persistence of the channel state due to slow fading, which is a departure from [16].

Additional information provides more and better transmission opportunities for the secondary. Unlike methods that limit the secondary operation to the ARQ re-transmission intervals in [16], the proposed method additionally allows the secondary to transmit elsewhere, for example, in any interval as long as it detects one of the following conditions: (a) the primary channel is so bad that the transmitter would fail to deliver the information after two transmissions regardless of secondary action or inaction, or (b) the primary channel is so good or cross channel is so weak that the secondary interference will not impede the primary even if it transmits at full power. Under both these conditions, we can allow the secondary to transmit without affecting the primary operations. Further transmit opportunities are also detected and exploited by a set of actions governed through a finite state machine at the secondary, which is described in the sequel.

The remainder of the paper is organized as follows. Section II introduces the system model. In Section III, two spectrum sharing schemes, namely aggressive and conservative SHARP which utilize the ACK/NAK feedback of the primary user, are proposed. Afterwards, the throughput analysis of the proposed schemes is given in Section IV. Finally, some simulation results and conclusion remarks are presented in Section $\mathrm{V}$ and VI, respectively.

\section{System MOdEL}

We consider a transmission system as shown in Figure 1. The primary transmitter occupies the channel at all time, therefore the secondary can use the channel only through spectrum sharing. The channel gains are shown by $g_{i j}$ from transmitter $i$ to receiver $j$, where the subscript value 1 denotes the primary and 2 denotes the secondary. Channel gains obey the exponential distribution with mean $\lambda$.

This paper considers a slow fading scenario, where the channel gain is assumed to be approximately constant over several transmission intervals, but is subject to change over much larger time scales. The primary transmitter operates at a constant power $P_{p}$ and the nominal spectral efficiency of $R_{p}$ bits/sec/Hz. If the first transmission at this rate and power is not successful (indicated by a NACK from the primary receiver), the same packet is re-transmitted at the same power. ${ }^{1}$ The two packets are combined at the receiver. If after two transmissions still the packet cannot be decoded, the primary declares outage and moves on to the next packet. Most data have some deadline for their arrival, therefore an unlimited number of ARQs is often not allowed. For the simplicity of exposition, we undertake the case of two transmissions for the developments of this paper. The ideas and the analysis directly extend to any number of ARQs, but the number of various outcomes in the system grows with the number of allowed ARQs without any essentially new insights. Therefore we limit the analysis in this paper to two ARQ rounds.

Whenever the secondary is activated, it has a peak power constraint of $P_{s}$ and transmits at a nominal spectral efficiency of $R_{s}$ bits/sec/Hz. The secondary receiver does not generate ARQ feedback. The intermittent transmission of the secondary will be characterized by the throughput that is a direct function of its outage, to be calculated in the sequel.

The fading intervals in this paper are taken to be much longer than the transmission intervals, and the primary transmits with constant rate and power. This system model has been chosen to shed light on the opportunities available to an agile secondary when the primary does not adapt its power and rate quickly to the channel conditions. This may happen, e.g., with legacy primary systems and standards, as newer technology allows the production of more agile wireless nodes. The system model is a simplified one (e.g. block fading with fixed rate and power in each fading interval instead of gradually changing) to focus on the most important aspects of the ideas and for simplicity of analysis. The contribution of this paper, essentially, is to show pragmatic methods of achieving spectrum sharing in the context of well-known and widely-used communication protocols, i.e., the ARQ.

\section{ARQ-BASEd OpPORTUNistic Spectrum SHARING}

The basic premise of this work is to allow a secondary to share the primary channel without explicit access to the primary channel-state-information (CSI). Partial channel state information is obtained via the ARQ from primary receiver. Furthermore, due to the persistence of channel state information (due to slow fading), the secondary is able to exploit opportunities that were not available, e.g., in [16]. In particular, in our case not only retransmission rounds, but also the first transmission rounds are candidates for spectrum sharing.

The basic idea of SHARP is to exploit transmission opportunities for the secondary when possible, but also avoid driving the primary into outage (as a result of interference). Unfortunately, the channel states are not directly available to the secondary due to practical reasons. The secondary can only observe the ACK/NACK from the primary receiver.

\footnotetext{
${ }^{1}$ In this paper we assume in the second round the same packet is retransmitted (packet combining) resulting in simpler analytical expressions. Sending new parity bits in the ARQ round (code combining) is also possible but is not considered in this paper.
} 


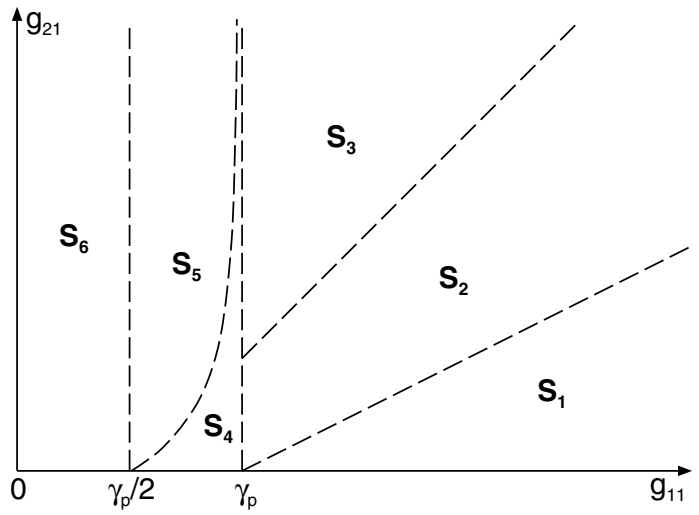

Fig. 2. The six regions for the operation of SHARP cognitive radio. For simplicity this figure assumes unit transmit powers and noise, i.e., $P_{p}=$ $P_{s}=N=1$.

The secondary also has knowledge of his own transmissions, therefore it can know whether the ACK/NACK of the primary was under the secondary interference or not.

The opportunity for activating the secondary depends on the relative strength of the direct channel $g_{11}$ and the cross channel $g_{21}$. We partition the $g_{11}-g_{21}$ plane into six regions, this partition is motivated by the amount of information that is available to the secondary, as will be shown in the sequel. We use the notations $\gamma_{p} \triangleq 2^{R_{p}}-1$ and $\gamma_{s} \triangleq 2^{R_{s}}-1$. The regions are characterized below and shown in Figure 2.

1) The primary channel supports the rate in one transmission despite secondary interference. This is the best of all worlds, the condition under which this is true can be characterized by:

$$
\frac{P_{p} g_{11}}{P_{s} g_{21}+N}>\gamma_{p}
$$

Under this condition, the secondary should always transmit.

2) The primary channel can support its rate in one transmission if there is no interference, but needs two transmissions to succeed if there is interference. This happens if:

$$
\begin{aligned}
\frac{\gamma_{p}}{2}<\frac{P_{p} g_{11}}{P_{s} g_{21}+N} & <\gamma_{p} \\
\frac{P_{p} g_{11}}{N} & >\gamma_{p}
\end{aligned}
$$

Under this condition, again the secondary can transmit at all times without pushing the primary into outage, but the throughput of the primary will be degraded.

3) The primary channel can support its rate in one transmission if there is no interference, but in the presence of interference it cannot succeed even with two transmissions:

$$
\begin{aligned}
\frac{P_{p} g_{11}}{P_{s} g_{21}+N} & <\frac{\gamma_{p}}{2} \\
\frac{P_{p} g_{11}}{N} & >\gamma_{p}
\end{aligned}
$$

Under this condition, the secondary can transmit every other time without causing outage for the primary, but the throughput of the primary will be degraded.
4) The primary channel can support its rate in two (but not one) interference-free transmissions; it can also succeed in two transmissions as long as only one of the two transmissions is subject to interference:

$$
\begin{array}{r}
\frac{P_{p} g_{11}}{N}+\frac{P_{p} g_{11}}{P_{s} g_{21}+N}>\gamma_{p} \\
\frac{\gamma_{p}}{2}<\frac{P_{p} g_{11}}{N}<\gamma_{p}
\end{array}
$$

Under this condition, the secondary should transmit only every other transmission interval without any effect on the primary.

5) The primary channel can support its rate in two (but not one) interference-free transmissions; it cannot support its rate with any interference (not even on one of its two transmissions).

$$
\begin{aligned}
\frac{P_{p} g_{11}}{N}+\frac{P_{p} g_{11}}{P_{s} g_{21}+N} & <\gamma_{p} \\
\frac{P_{p} g_{11}}{N} & >\frac{\gamma_{p}}{2}
\end{aligned}
$$

Under this condition, the secondary should remain silent.

6) If $g_{11}$ is sufficiently small, the primary is doomed to outage even with retransmission and even in the absence of any interference. This happens if:

$$
\frac{P_{p} g_{11}}{N}<\frac{\gamma_{p}}{2}
$$

Under this condition the secondary should transmit.

These six operating regions are denoted $S_{1}-S_{6}$ in Figure 2. We see that under some conditions, the secondary should stay silent to avoid pushing the primary into outage. Under some conditions, the secondary can transmit without any effect on the primary. Under some conditions, the secondary can transmit without causing outage to the primary, but it will slow down the primary and reduce its throughput because the primary will be forced to use re-transmissions.

Based on these observations, we can devise two algorithms. In the aggressive SHARP, the secondary will transmit whenever it is possible to do so without sending primary into outage, even if it will degrade the primary throughput. In the conservative SHARP, the secondary will only transmit when it has no effect on the primary.

We can now characterize the probing and discovery mechanism. The probing of the system is characterized by the secondary transmission decisions. For clarity, we use the following notation that combines the transmission modes of the primary and secondary.

$T_{0}=\{$ primary transmits new packet; secondary keeps silent $\}$ $T_{1}=\{$ primary repeats old packet; secondary keeps silent $\}$ $T_{2}=\{$ primary transmits new packet; secondary transmits $\}$ $T_{3}=\{$ primary repeats old packet; secondary transmits $\}$.

Using the above notation, the discovery mechanism for the secondary is relatively simple, and is shown in the flowcharts in Figures 3 and 4. The algorithm starts from the root of the tree, and proceeds to a leaf. Throughout this process, the secondary makes transmission decisions and observes the 
ACK/NACK from the primary, until it can determine which of the six regions it is operating in. The probing and channel detection for each of the six operating regions is outlined below.

$\left(S_{1}\right)$ is detected by receiving one ACK, probing the primary channel $\left(T_{2}\right)$ and receiving another ACK. This indicates that primary channel supports the rate in one transmission despite any interference.

$\left(S_{2}\right)$ is detected by receiving the first ACK, then the secondary probing in two successive intervals $\left(T_{2}, T_{3}\right)$ and getting a NACK followed by an ACK. This indicates the primary channel supports its rate in one interference-free transmissions, but in the presence of interference needs two transmissions to succeed.

$\left(S_{3}\right)$ is detected by receiving an ACK, then probing in two successive intervals $\left(T_{2}, T_{3}\right)$ and receiving two NACKs. This indicates the primary channel supports the rate in one interference-free transmissions, but in the presence of interference it is in outage even with retransmission.

$\left(S_{4}\right)$ is detected when the following sequence happens: receiving an initial NACK (which, recall, was under no interference), and the secondary staying silent and receiving a ACK (now we know the primary will get through in two transmissions if left alone). On the next transmission the secondary stays silent but hears a NACK (as expected), the next time the secondary transmits $\left(T_{3}\right)$ and hears an ACK. This indicates the primary channel supports the rate in two (but not one) interference-free transmissions; it can also succeed in two transmissions as long as only one of the transmissions is subject to interference.

$\left(S_{5}\right)$ is detected by going through the same sequence as the case above, however, in the last stage instead of an ACK a NACK is received, showing that despite all care the secondary cannot transmit. This indicates the primary channel supports the rate in two (but not one) interference-free transmissions, and that it cannot support its rate with interference (even on one of its two transmissions).

$\left(S_{6}\right)$ is detected by the secondary staying silent for two transmission intervals. When two successive NACKs are received, it is known that the primary is in outage even in the absence of secondary.

The detection of the operating region for aggressive SHARP can be implemented in a systematic way as shown in Figure 3. Starting from the root of the tree, the secondary stays silent for the first transmission and observes the primary ACK/NACK. Each of the six detection cases mentioned above traces a path from the root of the tree to one of the six leaves of the tree.

The control diagram for conservative SHARP is shown in Figure 4. Recall that in the two regions $S_{2}, S_{3}$, secondary transmission will reduce the primary throughput, therefore conservative SHARP refrains from transmitting in these two regions. Thus, the two outcomes $S_{2}, S_{3}$ are merged into $S_{2}^{\prime}$ for efficient representation.

Remark 1: We have so far assumed the channel is under slow fading, therefore the secondary will detect the channel and will continue to operate according to its findings. Naturally even a slow fading channel will eventually change.

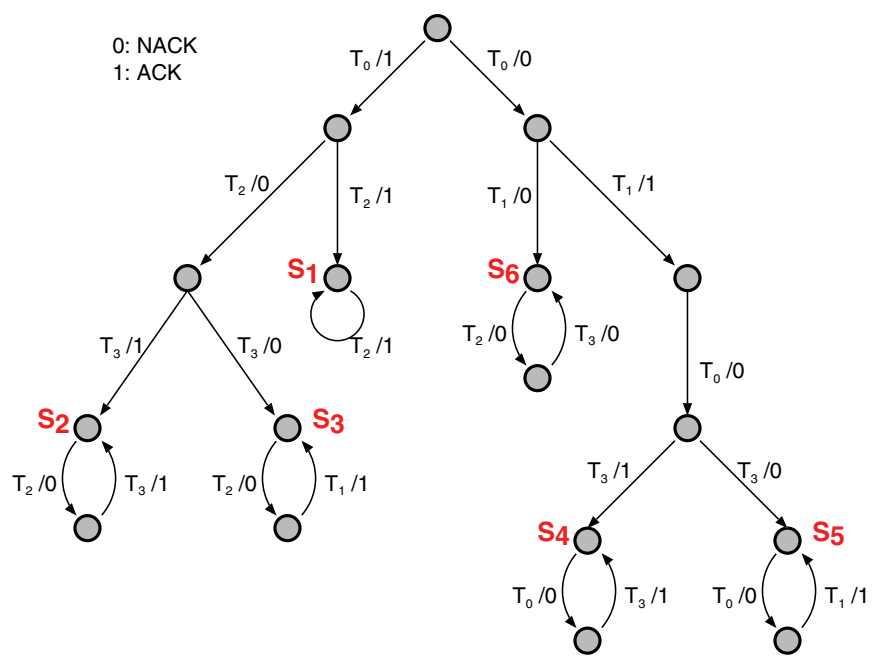

Fig. 3. Flow chart for the aggressive SHARP.

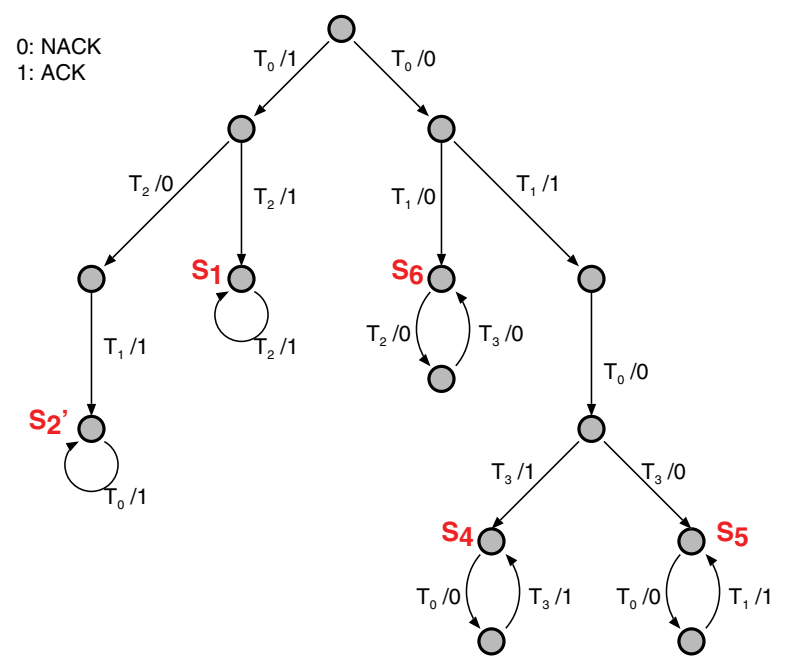

Fig. 4. Flow chart for the conservative SHARP. The outcomes $S_{2}, S_{3}$ from aggressive SHARP which resulted in primary throughput degradation are now modified into a single outcome $S_{2}^{\prime}$ where no secondary transmission is allowed.

This requires the secondary to periodically re-do the channel probing and re-assess the situation. In particular, each of the tree branches in our flow chart leads to a loop, which composes of one or two transmission modes from $T_{0}, T_{1}, T_{2}$, or $T_{3}$ and the corresponding ARQ feedbacks ( 1 or 0 ).

Remark 2: It should be noted that neither aggressive SHARP nor conservative SHARP produce any primary outage. The main difference between them is that aggressive SHARP may occasionally slow down the primary by forcing it to use two time slots instead of one. This is a slightly different form of degradation compared to [16], where the primary is slightly degraded in throughput because the secondary imposes on the primary (a small amount of) additional outage.

\section{Throughput AnAlysis}

We provide some analytical results in this section for the cognitive radio system when the aggressive or conservative SHARP scheme is applied. We first calculate the probabilities of the six channel regions. Then, we study the outage probability of primary and secondary. Finally, we derive the achievable 
throughput of the primary and secondary users. Note that we assume the Gaussian background noise variance $N$ equals to 1 for simplicity.

\section{A. Operating Region Probabilities}

The probability of the channel gains residing in each of the six operating regions shown in Figure 2 are as follows (Derivations in the Appendix):

$$
\begin{aligned}
P\left\{S_{1}\right\} & =P\left\{\frac{g_{11} P_{p}}{1+g_{21} P_{s}} \geq \gamma_{p}\right\}=\frac{\lambda_{11} P_{p} e^{-\frac{\gamma_{p}}{\lambda_{11} P_{p}}}}{\lambda_{21} P_{s} \gamma_{p}+\lambda_{11} P_{p}} \\
P\left\{S_{2}\right\} & =P\left\{\frac{g_{11} P_{p}}{1+g_{21} P_{s}}<\gamma_{p}, g_{11} P_{p} \geq \gamma_{p}, \frac{g_{11} P_{p}}{1+g_{21} P_{s}} \geq \frac{\gamma_{p}}{2}\right\} \\
& =\frac{\lambda_{21} P_{s} \gamma_{p} e^{-\frac{\gamma_{p}}{\lambda_{11} P_{p}}}}{\lambda_{21} P_{s} \gamma_{p}+\lambda_{11} P_{p}}-\frac{\lambda_{21} P_{s} \gamma_{p} e^{-\left(\frac{\gamma_{p}}{\lambda_{11} P_{p}}+\frac{1}{\lambda_{21} P_{s}}\right)}}{\lambda_{21} P_{s} \gamma_{p}+2 \lambda_{11} P_{p}}
\end{aligned}
$$$$
P\left\{S_{3}\right\}=P\left\{g_{11} P_{p} \geq \gamma_{p}, \frac{g_{11} P_{p}}{1+g_{21} P_{s}}<\frac{\gamma_{p}}{2}\right\}
$$$$
=\frac{\lambda_{21} P_{s} \gamma_{p} e^{-\left(\frac{\gamma_{p}}{\lambda_{11} P_{p}}+\frac{1}{\lambda_{21} P_{s}}\right)}}{\lambda_{21} P_{s} \gamma_{p}+2 \lambda_{11} P_{p}}
$$$$
P\left\{S_{4}\right\}=P\left\{g_{11} P_{p}+\frac{g_{11} P_{p}}{1+g_{21} P_{s}} \geq \gamma_{p}, g_{11} P_{p}<\gamma_{p}\right\}
$$$$
=e^{-\frac{\gamma_{p}}{\lambda_{11} P_{p}}}\left(\Phi\left(\lambda_{21} P_{s}, \frac{\lambda_{11} P_{p}}{\gamma_{p}}\right)-1\right)
$$$$
P\left\{S_{5}\right\}=P\left\{g_{11} P_{p} \geq \frac{\gamma_{p}}{2}, g_{11} P_{p}+\frac{g_{11} P_{p}}{1+g_{21} P_{s}}<\gamma_{p}\right\}
$$$$
=e^{-\frac{\gamma_{p}}{2 \lambda_{11} P_{p}}}-e^{-\frac{\gamma_{p}}{\lambda_{11} P_{p}}} \Phi\left(\lambda_{21} P_{s}, \frac{\lambda_{11} P_{p}}{\gamma_{p}}\right)
$$$$
P\left\{S_{6}\right\}=P\left\{g_{11} P_{p}<\frac{\gamma_{p}}{2}\right\}=1-e^{-\frac{\gamma_{p}}{2 \lambda_{11} P_{p}}}
$$

where $\Phi(y, z) \triangleq \int_{0}^{+\infty} e^{\frac{1}{(2+y t) z}-t} d t$.

The difference between aggressive and conservative SHARP is whether the secondary user is allowed to delay the primary's transmission. In the conservative scheme, Region $S_{2}$ and $S_{3}$ are combined to $S_{2}^{\prime}$ with

$$
\begin{aligned}
P\left\{S_{2}^{\prime}\right\} & =P\left\{\frac{g_{11} P_{p}}{1+g_{21} P_{s}}<\gamma_{p}, g_{11} P_{p} \geq \gamma_{p}\right\} \\
& =\frac{\lambda_{21} P_{s} \gamma_{p} e^{-\frac{\gamma_{p}}{\lambda_{11} P_{p}}}}{\lambda_{21} P_{s} \gamma_{p}+\lambda_{11} P_{p}}
\end{aligned}
$$

and the secondary user is not allowed to transmit in the region $S_{2}^{\prime}$.

\section{B. Outage Probability Analysis}

According to the description in Section III, there is no primary outage in Regions $S_{1}-S_{5}$, while in $S_{6}$ the primary is in outage. Therefore, the outage probabilities of the primary user for both aggressive and conservative SHARP are the same, and equal to $P\left\{S_{6}\right\}$ as given by (14).

In [16], the secondary user is allowed to transmit only when the interference from the primary is perfectly cancelled at the secondary receiver. The corresponding outage probability for the secondary is given as

$$
P^{\mathrm{OS}}=P_{\mathrm{PIC}}^{\mathrm{OS}}=1-e^{-\frac{\gamma_{s}}{\lambda_{22} P_{s}}} .
$$

where the superscript indicates the 'Outage for Secondary' and the subscript 'PIC' denotes 'Perfect Interference Cancellation.' The result is based on the assumption that the secondary receiver is located either far away or close to the primary transmitter.

In this paper, we take the interference into the consideration, and allow the secondary to coexist with the interference from the primary user. Specifically, the secondary tries to cancel the interference from primary in the first place. If the received signal-to-interference-plus-noise ratio (SINR) is greater than $\gamma_{p}$, the interference is considered to be cancelled from the received signal. Otherwise, the secondary simply treats the interference as the additional background noise. In this method the secondary receiver attempts to cancel interference signals using only the information received in one block, hence this approach is denoted single-block interference cancellation. The outage probability of the secondary is then given as

$$
\begin{aligned}
P^{\mathrm{OS}}= & P_{\mathrm{SBIC}}^{\mathrm{OS}}=P\left\{\frac{g_{12} P_{p}}{1+g_{22} P_{s}} \geq \gamma_{p}, g_{22} P_{s}<\gamma_{s}\right\} \\
& +P\left\{\frac{g_{12} P_{p}}{1+g_{22} P_{s}}<\gamma_{p}, \frac{g_{22} P_{s}}{1+g_{12} P_{p}}<\gamma_{s}\right\},
\end{aligned}
$$

where the first item denotes the case that the interference from the primary is perfectly cancelled at the secondary receiver, and the second term refers to the case that the primary interference is treated as the background noise. Moreover, (17) can be derived as

$$
\begin{aligned}
P^{\mathrm{OS}} & =1-e^{-\frac{\gamma_{s}}{\lambda_{22} P_{s}}}+\frac{e^{-\frac{\gamma_{s}}{\lambda_{22} P_{s}}}}{\left(\frac{\lambda_{22} P_{s}}{\lambda_{12} P_{p} \gamma_{s}}+1\right)}-\frac{e^{-\frac{\gamma_{s}}{\lambda_{22} P_{s}}-\frac{\gamma_{p}+\gamma_{p} \gamma_{s}}{\lambda_{12} P_{p}}}}{\left(\frac{\lambda_{22} P_{s} \gamma_{p}}{\lambda_{12} P_{p}}+1\right)} \\
& +I_{1} \cdot \mathbb{1}_{\gamma_{s} \gamma_{p}<1}
\end{aligned}
$$

where

$$
\begin{aligned}
I_{1}= & \frac{e^{-\frac{\gamma_{p}}{\lambda_{12} P_{p}}-\left(\frac{\lambda_{22} P_{s} \gamma_{p}}{\lambda_{12} P_{p}}+1\right) \frac{\gamma_{s}\left(1+\gamma_{p}\right)}{\lambda_{22} P_{s}\left(1-\gamma_{s} \gamma_{p}\right)}}}{\left(\frac{\lambda_{22} P_{s} \gamma_{p}}{\lambda_{12} P_{p}}+1\right)} \\
& -\frac{e^{\frac{1}{\lambda_{12} P_{p}}-\left(\frac{\lambda_{22} P_{s}}{\lambda_{12} P_{p} \gamma_{s}}+1\right) \frac{\gamma_{s}\left(1+\gamma_{p}\right)}{\lambda_{22} P_{s}\left(1-\gamma_{s} \gamma_{p}\right)}}}{\left(\frac{\lambda_{22} P_{s}}{\lambda_{12} P_{p} \gamma_{s}}+1\right)}
\end{aligned}
$$

and $\mathbb{1}_{\mathcal{A}}$ denotes the indicator function.

It has been noticed in [12] that the secondary receiver is able to buffer the initial received packet and attempts to cancel the interference from the primary as a whole when both copies in the two transmission slots are received, i.e. Backward Interference Cancellation (BIC). In this manner, the throughput of the secondary user can be further improved. The difference lies in the fact that $\mathrm{BIC}$ requires the secondary receiver to eavesdrop on the ARQ feedbacks from the primary receiver so that the decoder can recognize whether the interference from the primary is the repeating copy or a totally new packet. If this ARQ information is available at the secondary receiver, the outage probability for the secondary user can be further improved as follows:

$$
P^{\mathrm{OS}}= \begin{cases}P_{\mathrm{SBIC}}^{\mathrm{OS}} & S_{1} \\ P_{\mathrm{BIC} 1}^{\mathrm{OS}} & S_{2} \text { and } S_{6} \\ P_{\mathrm{BIC} 2}^{\mathrm{OS}} & S_{3} \text { and } S_{4} \\ \mathrm{~N} / \mathrm{A} & S_{5} \text { and } S_{2}^{\prime}\end{cases}
$$


where the detailed explanations are

- When in operating region $S_{1}$, the primary packet is sent only once from the primary transmitter. Therefore, (18) still holds.

- When in operating region $S_{2}$ and $S_{6}$, the primary packet is going to be repeated, and the secondary is able to buffer the primary message and try to decode it after receiving two copies of the same message. In the meanwhile, the secondary transmitter sends packets in both slots. The outage probability of this BIC scheme is

$$
\begin{aligned}
P_{\mathrm{BIC} 1}^{\mathrm{OS}}= & P\left\{\frac{2 g_{12} P_{p}}{1+g_{22} P_{s}} \geq \gamma_{p}, g_{22} P_{s}<\gamma_{s}\right\} \\
& +P\left\{\frac{2 g_{12} P_{p}}{1+g_{22} P_{s}}<\gamma_{p}, \frac{g_{22} P_{s}}{1+g_{12} P_{p}}<\gamma_{s}\right\} \\
= & 1-e^{-\frac{\gamma_{s}}{\lambda_{22} P_{s}}}+\frac{e^{-\frac{\gamma_{s}}{\lambda_{22} P_{s}}}}{\left(\frac{\lambda_{22} P_{s}}{\lambda_{12} P_{p} \gamma_{s}}+1\right)} \\
& -\frac{e^{-\frac{\gamma_{s}}{\lambda_{22} P_{s}}-\frac{\gamma_{p}+\gamma_{p} \gamma_{s}}{2 \lambda_{12} P_{p}}}}{\left(\frac{\lambda_{22} P_{s} \gamma_{p}}{2 \lambda_{12} P_{p}}+1\right)}+I_{2} \cdot \mathbb{1}_{\gamma_{s} \gamma_{p}<2}
\end{aligned}
$$

where

$$
\begin{aligned}
& I_{2}= \frac{e^{-\frac{\gamma_{p}}{2 \lambda_{12} P_{p}}-\left(\frac{\lambda_{22} P_{s} \gamma_{p}}{2 \lambda_{12} P_{p}}+1\right) \frac{\gamma_{s}\left(2+\gamma_{p}\right)}{\lambda_{22} P_{s}\left(2-\gamma_{s} \gamma_{p}\right)}}}{\left(\frac{\lambda_{22} P_{s} \gamma_{p}}{2 \lambda_{12} P_{p}}+1\right)} \\
&-\frac{e^{\frac{1}{\lambda_{12} P_{p}}-\left(\frac{\lambda_{22} P_{s}}{\lambda_{12} P_{p} \gamma_{s}}+1\right) \frac{\gamma_{s}\left(2+\gamma_{p}\right)}{\lambda_{22} P_{s}\left(2-\gamma_{s} \gamma_{p}\right)}}}{\left(\frac{\lambda_{22} P_{s}}{\lambda_{12} P_{p} \gamma_{s}}+1\right)} .
\end{aligned}
$$

The result is derived by simply substituting all $\gamma_{p}$ in (18) with $\frac{\gamma_{p}}{2}$.

- When in operating region $S_{3}$ and $S_{4}$, the primary packet is sent twice, but the secondary utilizes the transmission slot once (either the first as in $S_{3}$ or the second as in $S_{4}$ ). Therefore, the corresponding outage probability with BIC can be obtained as

$$
\begin{aligned}
& P_{\mathrm{BIC} 2}^{\mathrm{OS}}=P\left\{g_{12} P_{p}+\frac{g_{12} P_{p}}{1+g_{22} P_{s}} \geq \gamma_{p}, g_{22} P_{s}<\gamma_{s}\right\} \\
& +P\left\{g_{12} P_{p}+\frac{g_{12} P_{p}}{1+g_{22} P_{s}}<\gamma_{p}, \frac{g_{22} P_{s}}{1+g_{12} P_{p}}<\gamma_{s}\right\} \\
& =1-e^{-\frac{\gamma_{s}}{\lambda_{22} P_{s}}}+\frac{e^{\frac{1}{\lambda_{12} P_{p}}}}{\frac{\lambda_{22} P_{s}}{\gamma_{s} \lambda_{12} P_{p}}+1} I_{3} \\
& -e^{-\frac{\gamma_{p}}{\lambda_{12} P_{p}}} \int_{\frac{\gamma_{s}}{\lambda_{22} P_{s}}}^{\frac{\gamma_{s}^{\prime}}{\lambda_{22} P_{s}}} e^{\frac{1}{2+\lambda_{22} P_{s} y} \frac{\gamma_{p}}{\lambda_{12} P_{p}}-y} d y
\end{aligned}
$$

where

$$
I_{3}=e^{-\left(\frac{\lambda_{22} P_{s}}{\gamma_{s} \lambda_{12} P_{p}}+1\right) \frac{\gamma_{s}}{\lambda_{22} P_{s}}}-e^{-\left(\frac{\lambda_{22} P_{s}}{\gamma_{s} \lambda_{12} P_{p}}+1\right) \frac{\gamma_{s}^{\prime}}{\lambda_{22} P_{s}}}
$$

and $\gamma_{s}^{\prime} \triangleq\left(\gamma_{s}+\gamma_{p} \gamma_{s}-2+\sqrt{\left(1+\gamma_{p}\right)^{2} \gamma_{s}^{2}+4 \gamma_{s}+4}\right) / 2$ which can be further proved to be greater than $\gamma_{s}$ through simple algebra.

- When in operating region $S_{5}$ and $S_{2}^{\prime}$ (for conservative SHARP), the secondary transmitter will remain silent..

\section{Throughput Analysis}

In this subsection, the effective throughput of the aggressive and conservative SHARP schemes are studied. As shown in Figure 3 , the primary packet is sent by only one transmission cycle in Region $S_{1}$ and two cycles in the other SNR regions.. Except in Region $S_{6}$, the packet is successfully decoded at the primary receiver. As a result, the throughput of the primary user for the aggressive SHARP is given as

$$
G_{p}^{\mathrm{A}}=R_{p} P\left\{S_{1}\right\}+\frac{R_{p}}{2} \sum_{i=2}^{5} P\left\{S_{i}\right\}
$$

where the superscript ' $\mathrm{A}$ ' denotes the aggressive SHARP and $\frac{R_{p}}{2}$ is due to the two consecutive transmission cycles.

Accordingly, the throughput of the secondary user in $a g$ gressive SHARP can be derived, following the flow chart in Figure 3, as follows

$$
\begin{aligned}
G_{s}^{\mathrm{A}}= & \left(R_{s}\left(P\left\{S_{1}\right\}+P\left\{S_{2}\right\}+P\left\{S_{6}\right\}\right)\right. \\
& \left.+\frac{R_{s}}{2}\left(P\left\{S_{3}\right\}+P\left\{S_{4}\right\}\right)\right)\left(1-P^{\mathrm{OS}}\right) .
\end{aligned}
$$

As described in Section III, apart from exploiting the transmission opportunities in $S_{1}$ and $S_{4}$ which makes no harm to the primary system, the secondary user slows down the primary by forcing it to use two transmission cycles in Region $S_{2}$ and $S_{3}$. Therefore, extra throughput gain over the conservative SHARP can be found through item $R_{s} P\left\{S_{2}\right\}+\frac{R_{s}}{2} P\left\{S_{3}\right\}$ in (23). In addition, the secondary is allowed to transmit when two interference-free transmissions are not good enough to support the primary (Region $S_{6}$ ).

The conservative SHARP aims to avoid any negative effect on the primary user by allowing the secondary to transmit only when the channel is good enough to support simultaneous communication for both the primary and the secondary. The conservative scheme precludes transmission in the region $S_{2}^{\prime}$ (i.e., $S_{2} \cup S_{3}$ ), and leaves the primary alone. Consequently, the throughput of the primary and the secondary in the conservative SHARP are given by

$$
G_{p}^{\mathrm{C}}=R_{p}\left(P\left\{S_{1}\right\}+P\left\{S_{2}^{\prime}\right\}\right)+\frac{R_{p}}{2}\left(P\left\{S_{4}\right\}+P\left\{S_{5}\right\}\right)
$$

$$
G_{s}^{\mathrm{C}}=\left(R_{s} P\left\{S_{1}\right\}+\frac{R_{s}}{2} P\left\{S_{4}\right\}+R_{s} P\left\{S_{6}\right\}\right)\left(1-P^{\mathrm{OS}}\right)
$$

For the scheme in [16], we can derive the throughput of the primary and the secondary as follows

$$
\begin{aligned}
& G_{p}^{\mathrm{L}}=R_{p}\left(P\left\{S_{1}\right\}+P\left\{S_{2}^{\prime}\right\}\right)+\frac{R_{p}}{2} P\left\{S_{4}\right\} \\
& G_{s}^{\mathrm{L}}=\frac{R_{s}}{2}\left(P\left\{S_{4}\right\}+P\left\{S_{5}\right\}\right)\left(1-P^{\mathrm{OS}}\right)
\end{aligned}
$$

where the superscript ' $L$ ' represents the legacy scheme [16]. Note that the primary throughput is lost by $\frac{R_{p}}{2} P\left\{S_{5}\right\}$ compared to (24). This is due to the possible outage in Region $S_{5}$ when the secondary user is activated.

It can be noticed that the throughput of the secondary user can be further improved by judiciously selecting the outage threshold $\gamma_{s}$ when the interference from the primary can be perfectly cancelled as in (16). The probabilities of $S_{1}$ to $S_{6}$ are 
basically irrelevant with the SINR threshold of the secondary. Therefore, the task here is equivalent to maximizing $T_{s} \triangleq$ $R_{s}\left(1-P^{\mathrm{OS}}\right)$, where the maximal throughput can be achieved by taking the first order derivative with respect to the SINR outage threshold, and the optimal $\gamma_{s}$ can be represented by

$$
\gamma_{s}^{*}=\frac{\lambda_{22} P_{s}}{\mathcal{W}_{0}\left(\lambda_{22} P_{s}\right)}-1 .
$$

where function $\mathcal{W}_{0}$ denotes the single-valued Lambert $\mathrm{W}$ Function [17] for $W \geq-1$. Due to the complexity of (18) and (19), we can only derive the optimal outage threshold numerically.

\section{Numerical Results}

In this section, we present some numerical results of the proposed SHARP schemes. As stated in Section II, all the channels (primary, secondary, and two cross-interfering links) are assumed to follow the Rayleigh distribution. Unless otherwise notified, the constant power for the primary and secondary transmitter is equal to 1 as we did to the background noise in Section IV.

At the start, we study the scenario with weak interference from the secondary transmitter to the primary receiver. The mean of the channel propagation gains are $\lambda_{11}=4$, $\lambda_{21}=1$, and $\lambda_{22}=4$. The rate thresholds are set to be $R_{p}=1$ (bits $\left./ \mathrm{sec} / \mathrm{Hz}\right)$ and $R_{s}=0.5$ (bits $\left./ \mathrm{sec} / \mathrm{Hz}\right)$ for the primary and secondary user, respectively. Figure 5 shows the probabilities of the channel state falling in each Region as shown in Figure 2, when the secondary user transmit power $P_{s}$ increases from $-5 \mathrm{~dB}$ to $5 \mathrm{~dB}$. The markers symbolize the Monte Carlo simulation results (same afterwards), and the curves are obtained from the analytical formulae in (9)(14). It is demonstrated that the analytical results match the simulation results perfectly, which validates the correctness of the analytical results given in (9)-(14). In Figure 5, the probability of Region $S_{6}$ is a constant due to the fact that it is independent of secondary transmit power $P_{s}$. Moreover, it can be seen that the probabilities of Region $S_{1}$ and $S_{4}$ both decrease as $P_{s}$ increases.

Figure 6 shows the achievable throughput of the primary and the secondary for various SHARP schemes compared to the legacy scheme in [16] with the same parameters. Similar to the previous literature, the effective throughput of both parties is based on the mutual information outage provided in Section IV. It can be seen that the conservative SHARP is superior to the scheme in [16] in terms of throughput for both the primary and the secondary user. This is because in conservative SHARP the secondary is restricted from transmitting as long as it will jeopardize the primary transmission. Therefore, it avoids the possible primary outage in Region $S_{5}$, which may occasionally happen in the legacy scheme. Moreover, the conservative SHARP scheme adopts extra transmission opportunities in Region $S_{1}$ and $S_{6}$ which are not considered in [16], so the throughput of the secondary user increases dramatically. This increase can be further enhanced if the aggressive scheme is applied as shown in Figure 6. In this case, the secondary deliberately slows down the primary transmission in both $S_{2}$ and $S_{3}$ when the primary transmission could be successful within two transmission slots. As a result, the throughput of

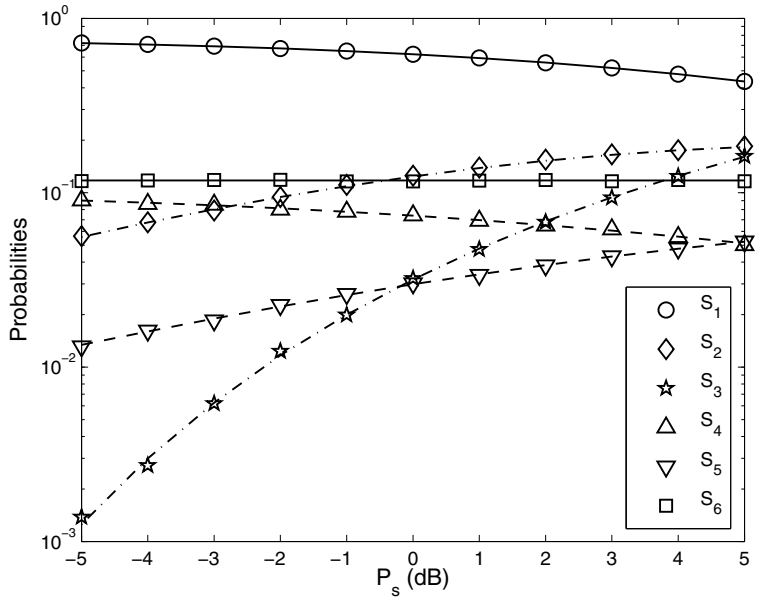

Fig. 5. Probability for six regions $\left[R_{p}=1, P_{p}=N=1, \lambda_{11}=\lambda_{22}=4\right.$, $\left.\lambda_{21}=1\right]$

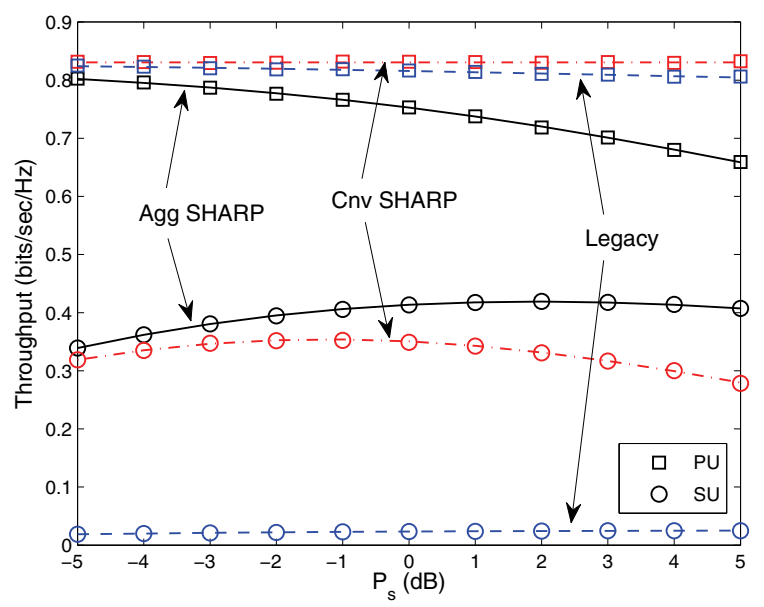

Fig. 6. Throughput for SHARP schemes $\left[R_{p}=1, R_{s}=0.5, P_{p}=N=1\right.$, $\lambda_{11}=\lambda_{22}=4, \lambda_{21}=1$, PU: primary user, SU: secondary user].

the primary user is degraded compared to the conservative scheme. It can be also observed that the secondary throughput of the conservative SHARP decreases when $P_{s}$ increases. This is due to the decrease of the probabilities of Regions $S_{1}$ and $S_{4}$ as shown in Figure 5.

Figure 7 illustrates how the throughput evolves with the signal to interference ratio (SIR) at the primary receiver, where we keep the transmission power (both PU and SU) and the channel gain for the primary link the same, and vary the interfering channel from the secondary to the primary. Notice that the optimal rate threshold for the secondary is derived from (28). The graph shows the similar comparison results among three schemes. In addition, the conservative SHARP makes no throughput loss to the primary as promised in Section III, while keeping the secondary throughput increasing when there is less interference at the primary receiver. The same tendency can also be observed for aggressive SHARP where both primary's and the secondary's throughput increase with weaker interfering channel. Higher SIR has also benefited 


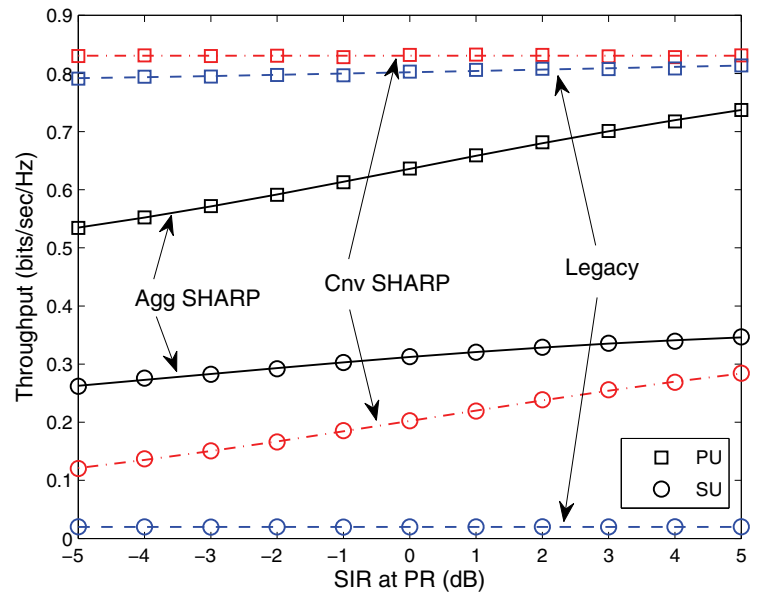

Fig. 7. Throughput for SHARP schemes $\left[R_{p}=1, P_{p}=P_{s}=N=1\right.$, $\lambda_{11}=4, \lambda_{22}=1$, PU: primary user, SU: secondary user].

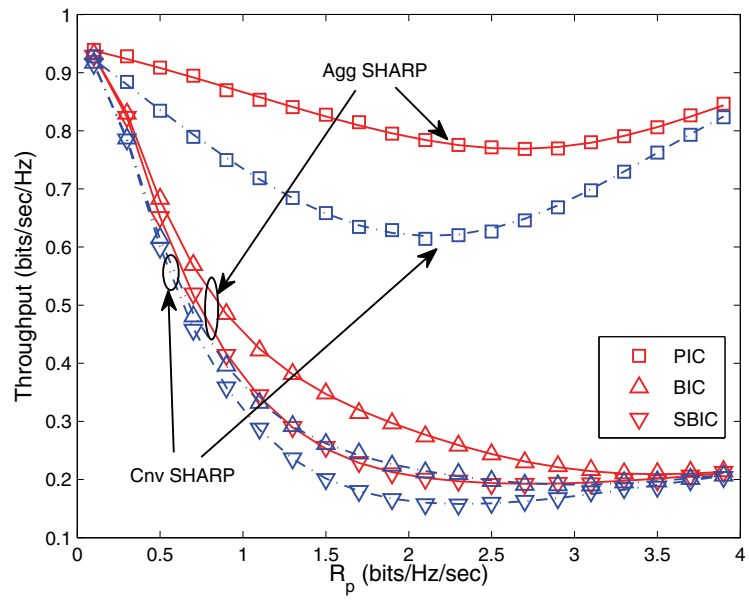

Fig. 8. Secondary user throughput with different interference cancellation schemes $\left[R_{s}=2, P_{p}=P_{s}=N=1, \lambda_{11}=\lambda_{22}=\lambda_{12}=4, \lambda_{21}=1\right]$.

the primary in the legacy system in that the probability of $S_{4}$ in (26) increases with small amount.

Figure 8 provides the secondary user throughput results with three interference cancellation schemes in Section IV-B. Both aggressive and conservative SHARP are considered and denoted by solid and dash-dot lines, respectively. We vary the rate outage threshold for the primary and observe that all three schemes are similar to each other in terms of the secondary user throughput when the rate threshold $R_{p}$ is low, but the single-block interference cancellation scheme has some difficulties when the requirement for decoding gets higher. If assuming the availability of ARQ feedbacks at the secondary receiver, backward interference cancellation can even improve the throughput performance for the secondary user. Note that throughput with all three schemes increase a little bit when $R_{p}$ is too high. This is because the probability of $S_{6}$ is higher and the secondary user has more opportunities to transmit in both two timeslots.

\section{CONCLUSION}

This paper proposes a transmission scheme, namely SHARP, for the secondary user co-existing with an ARQbased primary system. Based on the ACK/NAK message from the primary only, the proposed SHARP schemes utilize several probing time slots to obtain a general picture about the primary channel condition, and operate accordingly with suitable transmission modes. The proposed schemes have been analyzed and validated through Monte Carlo simulations. Furthermore, it was demonstrated that the aggressive SHARP achieves a better throughput than the conservative scheme with a small primary throughput loss. The conservative SHARP makes no negative effect on the primary system, and performs even better than the legacy system in terms of the primary user throughput. Both SHARP schemes do not generate unnecessary outage to the primary system, and are able to provide dramatic throughput gains to the secondary user without perfect CSI at the transmitter side.

\section{APPENDIX}

For the sake of simplicity, we assume the background noise equals to 1 , and decouple the channel gain by the mean value $\lambda$ and random variable $x$, i.e. $g_{11} \triangleq \lambda_{11} x_{11}$ and $g_{21} \triangleq \lambda_{21} x_{21}$, where $x_{11}, x_{21}$ follow exponential distribution with unit mean. Such decompositions are common in wireless communication for convenience of analysis in order to isolate and normalize the effect of small scale fading.

First, the probability of Region $S_{1}$ can be easily derived as

$$
\begin{aligned}
P\left\{S_{1}\right\} & =P\left\{\frac{g_{11} P_{p}}{1+g_{21} P_{s}} \geq \gamma_{p}\right\} \\
& =P\left\{x_{11} \geq \frac{\gamma_{p}\left(1+\lambda_{21} P_{s} x_{21}\right)}{\lambda_{11} P_{p}}\right\} \\
& =\int_{0}^{+\infty} e^{-\frac{\gamma_{p}\left(1+\lambda_{21} P_{s} y\right)}{\lambda_{11} P_{p}}} e^{-y} d y \\
& =\frac{\lambda_{11} P_{p} e^{-\frac{\gamma_{p}}{\lambda_{11} P_{p}}}}{\lambda_{21} P_{s} \gamma_{p}+\lambda_{11} P_{p}} .
\end{aligned}
$$

The probability of the combined Region $S_{2}^{\prime}=S_{2} \cup S_{3}$ is

$$
\begin{aligned}
P\left\{S_{2}^{\prime}\right\} & =P\left\{g_{11} P_{p} \geq \gamma_{p}, \frac{g_{11} P_{p}}{1+g_{21} P_{s}}<\gamma_{p}\right\} \\
& =P\left\{\frac{\gamma_{p}}{\lambda_{11} P_{p}} \leq x_{11}<\frac{\gamma_{p}\left(1+\lambda_{21} P_{s} x_{21}\right)}{\lambda_{11} P_{p}}\right\} \\
& =\int_{0}^{+\infty}\left[e^{-\frac{\gamma_{p}}{\lambda_{11} P_{p}}}-e^{-\frac{\gamma_{p}\left(1+\lambda_{21} P_{s} y\right)}{\lambda_{11} P_{p}}}\right] e^{-y} d y \\
& =\frac{\lambda_{21} P_{s} \gamma_{p} e^{-\frac{\gamma_{p}}{\lambda_{11} P_{p}}}}{\lambda_{21} P_{s} \gamma_{p}+\lambda_{11} P_{p}},
\end{aligned}
$$

where the probability of $S_{3}$ is

$$
\begin{aligned}
P\left\{S_{3}\right\} & =P\left\{g_{11} P_{p} \geq \gamma_{p}, \frac{g_{11} P_{p}}{1+g_{21} P_{s}}<\frac{\gamma_{p}}{2}\right\} \\
& =P\left\{\frac{\gamma_{p}}{\lambda_{11} P_{p}} \leq x_{11}<\frac{\gamma_{p}\left(1+\lambda_{21} P_{s} x_{21}\right)}{2 \lambda_{11} P_{p}}, x_{21}>\frac{1}{\lambda_{21} P_{s}}\right\} \\
& =\int_{\frac{1}{\lambda_{21} P_{s}}}^{+\infty}\left[e^{-\frac{\gamma_{p}}{\lambda_{11} P_{p}}}-e^{-\frac{\gamma_{p}\left(1+\lambda_{21} P_{s} y\right)}{2 \lambda_{11} P_{p}}}\right] e^{-y} d y
\end{aligned}
$$




$$
\begin{aligned}
& =\left.\left[e^{-\frac{\gamma_{p}}{\lambda_{11} P_{p}}} \frac{e^{-y}}{-1}-\frac{e^{-\frac{\gamma_{p}}{2 \lambda_{11} P_{p}}-\frac{\gamma_{p}}{2 \lambda_{11} P_{p}}-\frac{1}{\lambda_{21} P_{s}}}}{-\left(\frac{\gamma_{p} \lambda_{21} P_{s}}{2 \lambda_{11} P_{p}}+1\right)}\right]\right|_{y=\frac{1}{\lambda_{21} P_{s}}} ^{+\infty} \\
& =\frac{\lambda_{21} P_{s} \gamma_{p} e^{-\left(\frac{\gamma_{p}}{\lambda_{11} P_{p}}+\frac{1}{\lambda_{21} P_{s}}\right)}}{\lambda_{21} P_{s} \gamma_{p}+2 \lambda_{11} P_{p}}
\end{aligned}
$$

and consequently

$$
\begin{aligned}
P\left\{S_{2}\right\} & =P\left\{S_{2}^{\prime}\right\}-P\left\{S_{3}\right\} \\
& =\frac{\lambda_{21} P_{s} \gamma_{p} e^{-\frac{\gamma_{p}}{\lambda_{11} P_{p}}}}{\lambda_{21} P_{s} \gamma_{p}+\lambda_{11} P_{p}}-\frac{\lambda_{21} P_{s} \gamma_{p} e^{-\left(\frac{\gamma_{p}}{\lambda_{11} P_{p}}+\frac{1}{\lambda_{21} P_{s}}\right)}}{\lambda_{21} P_{s} \gamma_{p}+2 \lambda_{11} P_{p}} .
\end{aligned}
$$

Similarly, we can derive probability of Region $S_{4} \cup S_{5}$ in the first place

$$
\begin{aligned}
P\left\{S_{4} \cup S_{5}\right\} & =P\left\{g_{11} P_{p} \geq \frac{\gamma_{p}}{2}, g_{11} P_{p}<\gamma_{p}\right\} \\
& =e^{-\frac{\gamma_{p}}{2 \lambda_{11} P_{p}}}-e^{-\frac{\gamma_{p}}{\lambda_{11} P_{p}}} .
\end{aligned}
$$

and

$$
\begin{aligned}
P\left\{S_{5}\right\} & =P\left\{g_{11} P_{p} \geq \frac{\gamma_{p}}{2}, g_{11} P_{p}+\frac{g_{11} P_{p}}{1+g_{21} P_{s}}<\gamma_{p}\right\} \\
& =P\left\{\frac{\gamma_{p}}{2 \lambda_{11} P_{p}} \leq x_{11}<\left(\frac{1+\lambda_{21} P_{s} x_{21}}{2+\lambda_{21} P_{s} x_{21}}\right) \frac{\gamma_{p}}{\lambda_{11} P_{p}}\right\} \\
& =\int_{0}^{+\infty}\left[e^{-\frac{\gamma_{p}}{2 \lambda_{11} P_{p}}}-e^{\left.-\left(\frac{1+\lambda_{21} P_{s} t}{2+\lambda_{21} P_{s} t}\right) \frac{\gamma_{p}}{\lambda_{11} P_{p}}\right] e^{-t} d t}\right. \\
& =e^{-\frac{\gamma_{p}}{2 \lambda_{11} P_{p}}}-\int_{0}^{+\infty} e^{-\frac{\gamma_{p}}{\lambda_{11} P_{p}}+\frac{\gamma_{p}}{\left(2+\lambda_{21} P_{s} t\right) \lambda_{11} P_{p}}-t} d t \\
& =e^{-\frac{\gamma_{p}}{2 \lambda_{11} P_{p}}}-e^{-\frac{\gamma_{p}}{\lambda_{11} P_{p}}} \Phi\left(\lambda_{21} P_{s}, \frac{\lambda_{11} P_{p}}{\gamma_{p}}\right)
\end{aligned}
$$

As a result,

$$
\begin{aligned}
P\left\{S_{4}\right\} & =P\left\{S_{4} \cup S_{5}\right\}-P\left\{S_{5}\right\} \\
& =e^{-\frac{\gamma_{p}}{\lambda_{11} P_{p}}}\left(\Phi\left(\lambda_{21} P_{s}, \frac{\lambda_{11} P_{p}}{\gamma_{p}}\right)-1\right)
\end{aligned}
$$

where $\Phi(y, z) \triangleq \int_{0}^{+\infty} e^{\frac{1}{(2+y t) z}-t} d t$.

Finally, the probability of $S_{6}$ is given as

$$
P\left\{S_{6}\right\}=P\left\{g_{11} P_{p} \leq \frac{\gamma_{p}}{2}\right\}=1-e^{-\frac{\gamma_{p}}{2 \lambda_{11} P_{p}}} .
$$

\section{REFERENCES}

[1] M. Gastpar, "On capacity under receive and spatial spectrum-sharing constraints," IEEE Trans. Inf. Theory, vol. 53, no. 2, pp. 471-487, Feb. 2007.

[2] A. Ghasemi and E. S. Sousa, "Fundamental limits of spectrum-sharing in fading environments," IEEE Trans. Wireless Commun., vol. 6, no. 2, pp. 649-658, Feb. 2007.

[3] K. B. Letaief and W. Zhang, "Cooperative communications for cognitive radio networks," Proc. IEEE, vol. 97, no. 5, pp. 878-893, May 2009.

[4] Q. Zhang, J. Jia, and J. Zhang, "Cooperative relay to improve diversity in cognitive radio networks," IEEE Commun. Mag., vol. 47, no. 2, pp. 111-117, Feb. 2009.

[5] S. Srinivasa and S. A. Jafar, "The throughput potential of cognitive radio: a theoretical perspective," IEEE Commun. Mag., vol. 45, no. 5, pp. 73-79, May 2007.

[6] A. Goldsmith, S. A. Jafar, I. Maric, and S. Srinivasa, "Breaking spectrum gridlock with cognitive radios: an information theoretic perspective," Proc. IEEE, vol. 97, no. 5, pp. 894-914, May 2009.

[7] K. Eswaran, M. Gastpar, and K. Ramchandran, "Bits through ARQs: spectrum sharing with a primary packet system," in Proc. 2007 IEEE Int. Symp. on Information Theory, pp. 2171-2175.

[8] — - "Cognitive radio through primary control feedback," IEEE J. Sel. Areas Commun., vol. 29, no. 2, pp. 384-393, Feb. 2011.
[9] M. Levorato, U. Mitra, and M. Zorzi, "Cognitive interference management in retransmission-based wireless networks," in Proc. 2009 Allerton Conf. on Commun. Control and Computing, pp. 94-101.

[10] - "Cognitive interference management in retransmission-based wireless networks," IEEE Trans. Inf. Theory, vol. 58, no. 5, pp. 3023 3046, May 2012.

[11] N. Michelusi, O. Simeone, M. Levorato, P. Popovski, and M. Zorzi, "Optimal cognitive transmission exploiting redundancy in the primary ARQ process," in Proc. 2011 Information Theory and Applications Workshop, pp. 1-10.

[12] N. Michelusi, P. Popovski, M. Levorato, O. Simeone, and M. Zorzi, "Cognitive transmissions under a primary ARQ process via backward interference cancellation," in Proc. 2011 Allerton Conf. on Commun. Control and Computing, pp. 727-735.

[13] R. Zhang, "On active learning and supervised transmission of spectrum sharing based cognitive radios by exploiting hidden primary radio feedback," in Proc. 2009 IEEE Global Commun. Conf., pp. 1-5.

[14] R. Zhang, F. Gao, and Y.-C. Liang, "Cognitive beamforming made practical: effective interference channel and learning-throughput tradeoff," IEEE Trans. Commun., vol. 58, no. 2, pp. 706-718, Feb. 2010.

[15] S. Huang, X. Liu, and Z. Ding, "Decentralized cognitive radio control based on inference from primary link control information," IEEE J. Sel. Areas Commun., vol. 29, no. 2, pp. 394-406, Feb. 2011.

[16] R. A. Tannious and A. Nosratinia, "Cognitive radio protocols based on exploiting hybrid ARQ retransmissions," IEEE Trans. Wireless Commun., vol. 9, no. 9, pp. 2833-2841, Sep. 2010.

[17] R. M. Corless, G. H. Gonnet, D. E. G. Hare, D. J. Jeffrey, and D. E. Knuth, "On the Lambert W function," Adv. Comput. Math., vol. 5, pp. 329-359, Sep. 1996.

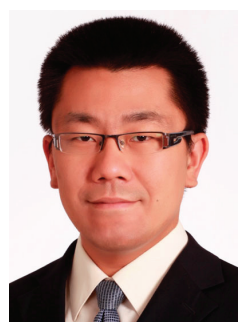

James C. F. Li was born in Beijing, China in 1979. $\mathrm{He}$ received his $\mathrm{Ph} . \mathrm{D}$. in Electrical and Electronic Engineering from The University of Melbourne in 2008. In Nov. 2009, he joined the School of Electrical Engineering and Telecommunications, The University of New South Wales, Sydney, Australia and worked as a research fellow. He is currently a research staff member at NEC Laboratories China in Beijing, where, after having worked on software defined radio and cognitive radio, he is currently investigating network-assisted device-to-device (D2D) communication and small cell associated issues. He has published more than 20 technical papers in the area of wireless communications and also acquired 10 pending or issued patents.

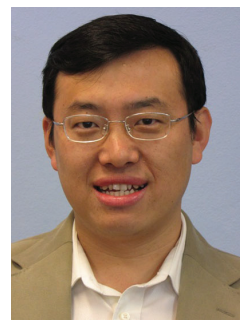

Wei Zhang (S'01-M'06-SM'11) received the Ph.D. degree in electronic engineering from The Chinese University of Hong Kong in 2005. He was a Research Fellow with the Department of Electronic and Computer Engineering, Hong Kong University of Science and Technology, during 2006-2007. In 2008, he joined the School of Electrical Engineering and Telecommunications, the University of New South Wales, Sydney, Australia, where he is currently an Associate Professor. His current research interests include cognitive radio, cooperative communications, space-time coding, and multiuser MIMO. He received the best paper award at the 50th IEEE Global Communications Conference (GLOBECOM), Washington DC, in 2007 and the IEEE Communications Society Asia-Pacific Outstanding Young Researcher Award in 2009. He is Co-Chair of Communications Theory Symposium of International Conference on Communications (ICC 2011), Kyoto, Japan. He is an Editor of the IEEE TRANSACTIONS ON WIRELESS COMMUNICATIONS and an Editor of the IEEE JOURNAL ON Selected Areas in Communications (Cognitive Radio Series). 


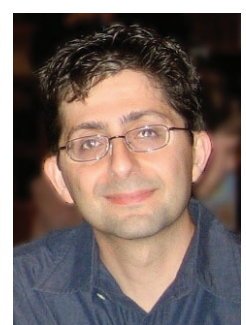

Aria Nosratinia (S'87-M'97-SM'04-F'10) is Erik Jonsson Distinguished Professor and associate head of the Electrical Engineering Department at the University of Texas at Dallas. He received his Ph.D. in Electrical and Computer Engineering from the University of Illinois at Urbana-Champaign in 1996. He has held visiting appointments at Princeton University, Rice University, and UCLA. His interests lie in the broad area of information theory and signal processing, with applications in wireless communications. He was the secretary of the IEEE Information Theory Society in 2010-2011 and the treasurer for ISIT 2010 in Austin, Texas. He is currently an area editor for IEEE TRANSACTIONS ON WIRELESS COMMUNICATIONS, and has in the past served as editor for the IEEE TRANSACTIONS ON INFORMATION THEORY, IEEE TRANSACTIONS on Wireless COMMUNiCATIONS, IEEE Signal PROCESSING LETTERS, IEEE Transactions on Image Processing, and IEEE Wireless Communications (Magazine). He has been a recipient of the National Science Foundation career award. He was elected fellow of IEEE for contributions to multimedia and wireless communication.

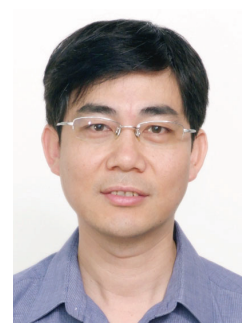

Jinhong Yuan received the B.E. and Ph.D. degrees in electronics engineering from Beijing Institute of Technology, Beijing, China, in 1991 and 1997, respectively. From 1997 to 1999 he was a Research Fellow at the School of Electrical Engineering, the University of Sydney, Sydney, Australia. In 2000 he joined the School of Electrical Engineering and Telecommunications, the University of New South Wales, Sydney, Australia, where he is currently a Professor for Telecommunications of the school. He has published two books, two book chapters, over 200 papers in telecommunications journals and conference proceedings and 40 industrial reports. He is a co-inventor of one patent on MIMO systems and two patents on low-density-parity-check (LDPC) codes. He coauthored three Best Paper Awards and one Best Poster Award, including a Best Paper Award of IEEE Wireless Communications and Networking Conference (WCNC), Cancun, Mexico in 2011, and a Best Paper Award of IEEE International Symposium on Wireless Communications Systems (ISWCS), Trondheim, Norway in 2007. His publication is available from http://www2.ee.unsw.edu.au/wcl/JYuan.html. He serves as the IEEE NSW Chair of joint Communications/Signal Processions/Ocean Engineering Chapter. His current research interests include error control coding and information theory, communication theory, and wireless communications. 